\title{
Power imbalance induced BER performance loss under limited-feedback CoMP techniques
}

\author{
Beneyam B. Haile ${ }^{1 *}$, Jyri Hämäläinen ${ }^{1}$ and Zhi Ding ${ }^{2}$
}

\begin{abstract}
Coordinated multipoint (CoMP) technology utilizes simultaneous transmission/reception from/to different access points, and it is considered as an important feature to exploit and/or mitigate intercell interference in fourth-generation mobile networks. Yet, channel power imbalance at the receiver is experienced in CoMP systems due to, e.g., spatially distributed transmissions. Traditional co-located multi-antenna systems may also experience power imbalance among antenna branches due to inaccurate antenna calibration. This paper presents a bit error rate (BER) analysis and derives asymptotic and approximate BER expressions for some practical CoMP transmission techniques under channel power imbalance. Besides the analytical results, numerical analysis is made to thoroughly capture the performance impact of channel power imbalance on the performance gain of the CoMP methods. The results demonstrate that power imbalance considerably affects BER performance and applying long-term amplitude information with fast phase feedback has insignificant benefit to effectively compensate the detrimental effect of large channel power imbalance when base stations use a single antenna. In this case, exploiting both short-term amplitude and phase information is a very good choice. On the contrary, for a large number of diversity antennas in base stations, using long-term amplitude information with a sparsely quantized phase shows BER performance close to the case where full channel state information is applied.
\end{abstract}

Keywords: Intercell interference, Coordinated multipoint, CoMP, LTE-advanced, Channel power imbalance, Transmit beamforming, Bit error rate

\section{Introduction}

The inconsistent quality of experience across mobile networks is an important challenge of contemporary mobile communications, the intercell interference being one of the main causes of the inconsistency [1]. Coordinated multipoint (CoMP) transmission has been recently proposed to mitigate and/or exploit intercell interference in mobile systems [2-6]. In CoMP, user data transmission is dynamically executed either from all coordinating base stations (BSs) or from one BS while scheduling/beamforming decisions are made together by coordinating BSs. CoMP transmission techniques have

*Correspondence: beneyam.haile@aalto.fi

${ }^{1}$ School of Electrical Engineering, Aalto University, P.O.Box 13000, FI-00076 Aalto, Espoo, Finland

Full list of author information is available at the end of the article also been standardized for 3rd Generation Partnership Project (3GPP) long-term evolution (LTE) [7, 8].

Various limited-feedback precoding methods with different implementation requirements for practical CoMP scenarios have been studied [9-12]. Most analytical studies rely on the conventional assumption that antenna branches are homogeneous and antenna channels seen by the receiver admit the same statistical properties. Yet, in many practical joint-transmission CoMP scenarios, mean power imbalance occurs among signals received by a user from different coordinating BSs. The power imbalance is due to either spatially distributed transmissions in the case of inter-site CoMP or different directions of sector antenna main lobes in the case of intra-site CoMP. Besides CoMP, a similar channel power imbalance problem occurs in distributed antenna systems where transmit antenna elements are geographically distributed but connected to the same controlling BS [13]. For instance, for 
a 3GPP urban micro pathloss model (i.e., $L=34.53+$ $38 \log _{10}(d)$ for a distance $d$ [14]), a mobile station (MS) located between two neighboring BS sites with an intersite distance of ISD experiences channel power imbalance of $\sigma_{d}=38 \log _{10}((\mathrm{ISD}+\Delta d) /(\mathrm{ISD}-\Delta d)) \mathrm{dB}$ due to distance difference $\triangle d$ from the sites. Also, for the 3GPP three-sector antenna pattern (i.e., $A(\theta)=-12\left(\theta / \theta_{3 \mathrm{~dB}}\right)^{2}$ for 3-dB beamwidth $\theta_{3 \mathrm{~dB}}$ [14]), a MS located between two neighboring co-located sectors at an angle of $\theta$ from the main lobe direction of one of the sectors experiences channel power imbalance of $\sigma_{\theta}=288(\theta-60) /\left(\theta_{3 \mathrm{~dB}}\right)^{2}$. An illustration for channel power imblance values is presented in Table 1 for ISD $=500 \mathrm{~m}$ and $\theta_{3 \mathrm{~dB}}=70^{\circ}$. Channel power imbalance also occurs among antenna branches in the conventional co-located multi-antenna systems due to imperfect antenna calibration. For example, in third-generation BS, the difference between reference signals from two different antenna connectors must be within $\pm 2 \mathrm{~dB}[15]$.

In [16], we presented a detailed analysis for the optimal amplitude weights, signal-to-noise ratio (SNR) gain, and average capacity under channel power imbalance in the case of selected CoMP techniques that are consistent with standardized limited-feedback methods. The paper [16] provides thorough insights on the performance loss due to power imbalance in terms of coherent combining gain. In this work, we study the impact of channel power imbalance on the bit error rate (BER) performance of the CoMP methods. To that end, we derive asymptotic and approximate BER expressions for the CoMP techniques assuming single-antenna BSs. As the analytical computations are cumbersome for the multi-antenna BSs, the channel power imbalance impact study in a more general case is made through simulation. For benchmarking purposes, we also recall BER results for transmitter selection combining and the case where full channel state information (CSI) is employed at the transmitter side. To the authors best knowledge, this BER performance analysis for the limited-feedback CoMP techniques has not been carried out in previous literature.

Our results illustrate how sensitive a limited-feedback technique that exploits long-term CSI feedback to maximize the SNR is to the channel power imbalance in the single-antenna case. We show the necessity of short-term

Table 1 Channel power imbalance values for a given $\triangle d$ or $\theta$ when ISD $=500 \mathrm{~m}$ and $\theta_{3 \mathrm{~dB}}=70^{\circ}$

\begin{tabular}{lll}
\hline$\sigma(\mathrm{dB})$ & $\Delta d(\mathrm{~m})$ & $\theta\left(^{\circ}\right)$ \\
\hline 0 & 0.0 & 60.0 \\
3 & 45.4 & 65.1 \\
6 & 90.0 & 70.2 \\
10 & 147.1 & 77.0 \\
\hline
\end{tabular}

amplitude feedback when channel power imbalance is large. Furthermore, we note that the short-term amplitude feedback is not important when a larger number of transmit antennas are employed in BSs.

In terms of organization, Section 2 provides an overview of the system model and the CoMP algorithms. In Section 3, we compute analytical expressions for the asymptotic and the approximate BERs when BSs use a single antenna. In Section 4, we verify analytical results and provide performance comparisons and simulation results when BSs apply more than one antenna. Finally, we present our conclusions in Section 5.

\section{System model and CoMP schemes}

\subsection{System Model}

The general system model is depicted in Fig. 1 where two groups of $M$ antenna branches transmit identical information to a single-antenna mobile station. Antenna groups can be located either in different sites as in the case of inter-site CoMP and distributed antenna systems or in the same site as in the case of intra-site CoMP. We note that CoMP involving two groups of antennas is an important joint transmission scenario that can mitigate/exploit the most dominant interference with the least overhead and complexity.

A feedback system with low-rate CSI from a singleantenna MS is used to select transmission weights for the antenna groups and branches in Fig. 1. In this model, the received signal at a given time instant can be written as

$$
r=\sum_{m=1}^{2} \mathbf{h}_{m} \cdot \mathbf{x}_{m}+n=\sum_{m=1}^{2}\left[\left(\mathbf{h}_{m} \cdot \mathbf{u}_{m}\right) w_{m}\right] s+n,
$$

where $\mathbf{x}_{m} \in \mathbb{C}^{1 \times M}$ is the transmitted vector signal from the $m$ th BS antennas containing the information symbol $s$ of the active user, $\mathbf{h}_{m} \in \mathbb{C}^{1 \times M}$ is the channel gain vector of the $m$ th group, and $n$ refers to zero-mean complex additive white Gaussian noise with power $P_{\mathrm{n}}$. We note that $\mathbf{x}_{m}$ comes from $s$ via beamforming, where $\mathbf{w}$ and $\mathbf{u}_{m}$ with normalized powers $\|\mathbf{w}\|=1$ and $\left\|\mathbf{u}_{m}\right\|=$ 1 represent complex weight vectors selected from given codebooks according to applied precoding techniques. The power constraint of the input signal implies that $\sum_{m=1}^{2} \mathbb{E}\left\{\mathbf{x}_{m}^{\dagger} \mathbf{x}_{m}\right\} \leq P_{\mathrm{t}}$, where $P_{\mathrm{t}}$ is the total transmitted energy per channel use and $(\cdot)^{\dagger}$ denotes Hermitian transpose. When $M=1,(2)$ is reduced to the form

$$
r=\left[h_{1,1} w_{1}+h_{2,1} w_{2}\right] s+n=(\mathbf{h} \cdot \mathbf{w}) s+n,
$$

where $\mathbf{h}=\left(h_{1,1}, h_{2,1}\right)$.

We consider a flat block fading channel model where channel gains remain stationary during each block of transmitted symbols, and channel responses from temporally separate transmission blocks are independent. Furthermore, the complex channel gains $h_{m, l}$ are assumed to 


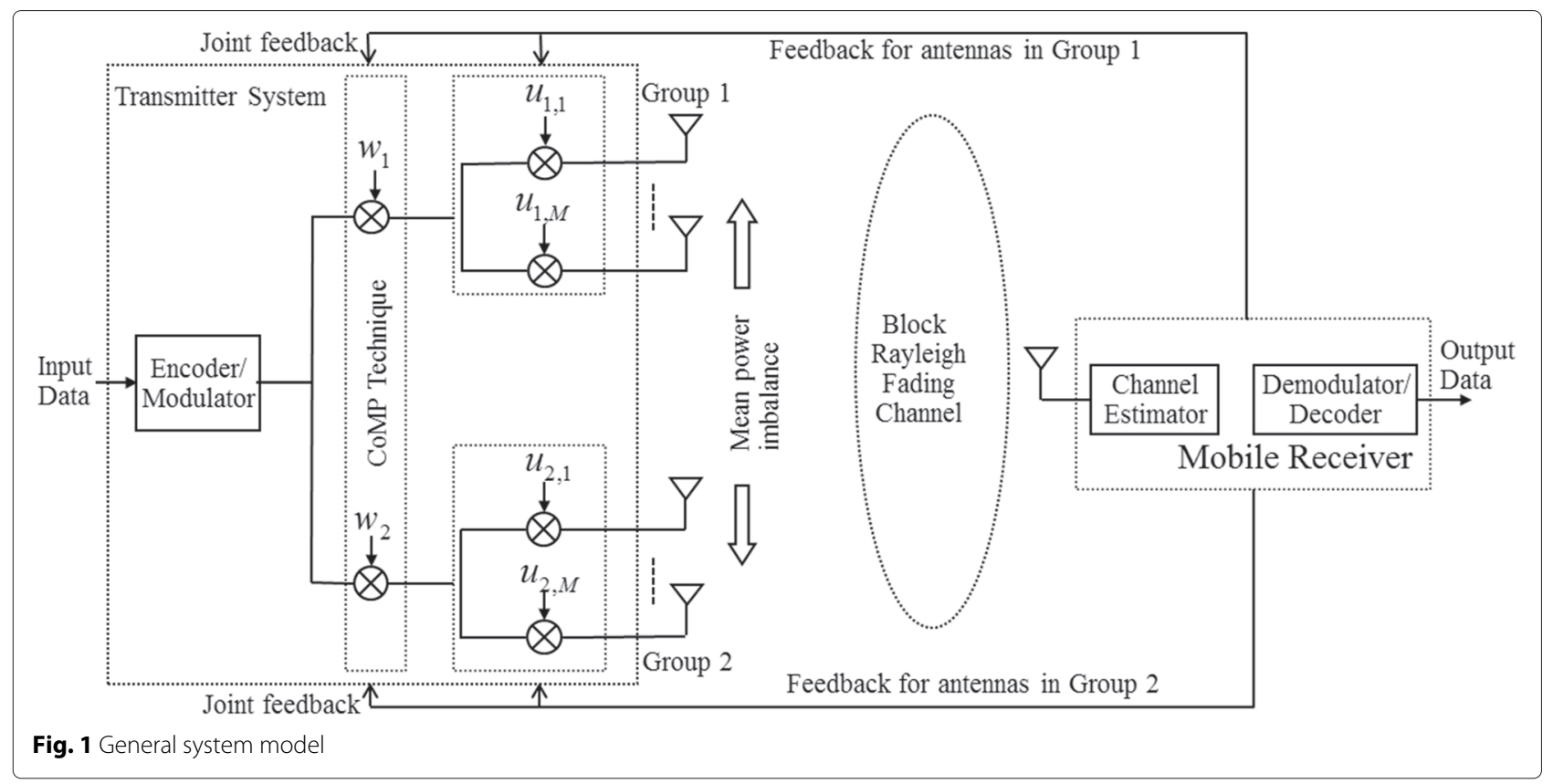

be independent and identically distributed zero-mean circularly symmetric complex Gaussian random variables: $h_{m, l}=\sqrt{\gamma_{m, l}} e^{j \psi_{m, l}}$. Hence, the branch power $\gamma_{m, l}$ follows exponential distribution with mean $\bar{\gamma}_{m}=\mathbb{E}\left\{\gamma_{m, l}\right\}$, and the branch phase $\psi_{m, l}$ is uniformly distributed on $(-\pi, \pi)$. Spatially uncorrelated channel assumption is considered as for the different groups we have antennas spatially well separated, and within the same group, both polarization and spatial separation of antennas can be effectively used especially in urban environments [17]. Of course, when the number of antennas becomes large, it will be increasingly difficult to obtain configuration where mutual correlation between antennas is small. We consider negligible power imbalance between antenna branches within the same group as they are co-located and directed in the same fashion and inexpensive calibration can be applied. We also assume that MS has perfect CSI and provides both short-term and long-term feedback to the distributed antenna transmitter. The short-term quantized CSI is available at the transmitter side without errors or latency while perfect time synchronization is assumed between transmission points such that coherent combining is possible. The long-term channel statistics is perfectly known at the transmitter side.

\subsection{CoMP algorithms}

This work investigates three joint processing CoMP algorithms. The algorithms are briefly defined to choose the best weight $\widehat{\mathbf{w}}$ when $M=1$. If $M>1$, the definitions are used by replacing $h_{m, 1}$ with the signal $\mathbf{h}_{m} \cdot \widehat{\mathbf{u}}_{m}$ where $\widehat{\mathbf{u}}_{m}$ is the best weight vector selected for the $m$ th antenna group [16].

\subsubsection{Transmitter selection combining (TSC)}

This is a simple classical method where the antenna branch providing the largest signal power is selected. Selection of better weight $\widehat{\mathbf{w}}$ is made according to $|\mathbf{h} \cdot \widehat{\mathbf{w}}|=$ $\max \left\{\left|h_{m, 1}\right|: m=1,2\right\}$. Only a 1-bit feedback overhead is required for this method which makes the algorithm attractive from an implementation perspective although its performance is inferior when compared to the more sophisticated CoMP algorithms, and it is also very sensitive to errors in feedback signaling [18]. TSC is included in the 3GPP CoMP category under the name dynamic point selection [7].

\subsubsection{Quantized co-phasing (QCP)}

In this CoMP scheme, MS reports the quantized relative phase of the channel gains. Thus, when using the $N_{w}$ bit feedback, we have

$$
|\mathbf{h} \cdot \widehat{\mathbf{w}}|=\max \left\{\left|h_{1,1} v_{1}+h_{2,1} v_{2} e^{j \phi_{n}}\right|: 1 \leq n \leq 2^{N_{w}}\right\},
$$

where $\phi_{n}=\pi n / 2^{N_{w}-1}$ and $v_{1}, v_{2}$ refer to selected transmit weights that determine the ratio of transmit power in each antenna group/branch. We select either equal weights $\left(v_{1}=v_{2}=\sqrt{1 / 2}\right)$ or long-term weights maximizing SNR based on the long-term CSI feedback [16]. If $N_{w}=2$ and there is no mean power imbalance between channels, then (3) resembles closed-loop transmit-diversity that is applied in 3GPP high-speed downlink packet access and LTE $[19,20]$. 


\subsubsection{Ordered quantized co-phasing (OQCP)}

This algorithm is a natural extension of QCP formed by using short-term order information of channel amplitudes in addition to the phase difference. In OQCP, the receiver first ranks instantaneous SNRs, $\left|h_{(1)}\right|=\max \left\{\left|h_{1,1}\right|,\left|h_{2,1}\right|\right\}$, and $\left|h_{(2)}\right|=\min \left\{\left|h_{1,1}\right|,\left|h_{2,1}\right|\right\}$ before deciding the phase feedback using criteria (3). Both order and phase difference information are signaled to the transmitter using $N_{w}+1$ feedback bits. After precoding, the received signal is of the form

$$
|\mathbf{h} \cdot \widehat{\mathbf{w}}|=\left|h_{(1)} v_{1}+h_{(2)} v_{2} e^{j \hat{\phi}_{n}}\right|,
$$

where $\hat{\phi}_{n}$ refers to the best phase and the normalized amplitude weights are selected based on the channel order statistics. In the case of Rayleigh fading under channel power balance, it has been shown that $v_{1}^{2}=(1+(1+$ $\left.\left.(\pi \rho / 2))^{2}\right)^{-1 / 2}\right) / 2, \rho=\operatorname{sinc}\left(\pi / 2^{N_{w}}\right)$ and $v_{2}^{2}=1-v_{1}^{2}[21]$.

If $M>1$, the best weight vector $\widehat{\mathbf{u}}_{m}$ is selected according to QCP. We let the first antenna branch of the $m$ th group as a reference, and the best phase for each remaining antenna branches is chosen and reported using $N_{u}$ feedback bits.

We note that although amplitude weights maximizing expected SNR are presented in [16] for both QCP and OQCP, the following BER analysis is valid for any transmit amplitude weights.

\section{Bit error rate analysis}

In the following, we characterize the impact of power imbalance on the average BER-denoted by $P_{e}-$ for $M=$ 1 . We start the analysis by recalling that the average BER can be computed from the formula

$$
P_{e}=\int_{0}^{\infty} P_{\bmod }(z) f(z) d z
$$

where $z=|\mathbf{h} \cdot \widehat{\mathbf{w}}|^{2}$ represents the instantaneous SNR when feedback algorithm is used and $P_{\bmod }(\cdot)$ is the error rate of the applied modulation scheme. For simplicity, we consider here only the BPSK modulation: $P_{\bmod }(z)=1 / 2$. $\operatorname{erfc}(\sqrt{z})$. It is also known that the symbol/bit error rates for the higher order modulation methods like M-QAM can be approximated or even in some cases expressed exactly by using the very same complementary error function $\operatorname{erfc}(\sqrt{z})$, after some simple scaling. Therefore, BER results for many modulation methods over the fading channel are easily obtained once BER has been computed for the BPSK modulation.

Closed-form expression for BER can be derived so long as the distribution $f(z)$ is known and (5) is analytically integrable. This is the case if perfect CSI is available or TSC is applied. On the other hand, for QCP and OQCP algorithms, the distribution $f(z)$ is difficult to obtain but we compute for both algorithms the asymptotic BER in closed form. We note that asymptotic analysis has been previously presented in [22] for the special case $\bar{\gamma}_{1}=\bar{\gamma}_{2}$. Then we formulate approximate BER expressions that can be used in the low-to-moderate SNR region based on the asymptotic BER expressions.

Although mean SNRs are not equal, we can assume that $\bar{\gamma}_{1}$ and $\bar{\gamma}_{2}$ grow at the same rate in the asymptotic SNR region. Then $\bar{\gamma}_{1} \bar{\gamma}_{2}=\sigma_{0} \bar{\gamma}_{1}^{2}$, where $\sigma_{0}$ denotes the channel power imbalance between the antenna branches. Now the asymptotic BER can be written in the form

$$
\log _{10} P_{e}\left(\bar{\gamma}_{1}, \bar{\gamma}_{2}\right) \approx \mathcal{E}\left(\sigma_{0}\right)-d \cdot \log _{10} \bar{\gamma}_{1}, \quad \bar{\gamma}_{1} \gg 1,(6)
$$

where the slope $d$ is the diversity gain. To validate the formula (6), we show that diversity gains of the investigated methods are equal to two despite the mean power imbalance. Furthermore, we deduce closed-form expressions for the constant $\mathcal{E}\left(\sigma_{0}\right)$ in the case of the CoMP methods of Section 2.

\subsection{Asymptotic BERs for reference methods}

If perfect CSI is available in the transmitter, then the distribution of received SNR is of the form $f(z)=$ $1 /\left(\bar{\gamma}_{1}-\bar{\gamma}_{2}\right)\left(e^{-z / \bar{\gamma}_{1}}-e^{-z / \bar{\gamma}_{2}}\right)$ [23] and the computation of BER can be carried out using (5). The result is well known, and with the aid of notation $\sigma_{0}$, we can express it as follows [24]:

$$
\begin{aligned}
& P_{e}= \\
& \frac{1}{2}\left[1-\frac{1}{1-\sigma_{0}} \sqrt{\frac{\bar{\gamma}_{1}}{1+\bar{\gamma}_{1}}}\left(1-\sigma_{0} \sqrt{\frac{\sigma_{0}\left(1+\bar{\gamma}_{1}\right)}{1+\sigma_{0} \bar{\gamma}_{1}}}\right)\right] .
\end{aligned}
$$

Furthermore, after utilizing Taylor series expansion on the square roots, we obtain the asymptotic formula

$$
\lim _{\bar{\gamma}_{1} \rightarrow \infty} \bar{\gamma}_{1}^{2} \cdot P_{e}=3 /\left(16 \cdot \sigma_{0}\right) \text {. }
$$

When comparing this formula with (6), we find that $d=$ 2 and $\mathcal{E}\left(\sigma_{0}\right)=\log _{10}\left(3 /\left(16 \sigma_{0}\right)\right)$.

The TSC has been well examined in the literature $[25,26]$. Using the adopted notations, the BER of TSC can be written in the form

$$
\begin{aligned}
& P_{e}= \\
& \frac{1}{2}\left[1-\sqrt{\frac{\bar{\gamma}_{1}}{1+\bar{\gamma}_{1}}}-\sqrt{\frac{\bar{\gamma}_{1} \sigma_{0}}{1+\bar{\gamma}_{1} \sigma_{0}}}+\sqrt{\frac{\bar{\gamma}_{1} \sigma_{0}}{1+\sigma_{0}\left(1+\bar{\gamma}_{1}\right)}}\right] .
\end{aligned}
$$

After applying the Taylor series expansion on the square roots, we obtain

$$
\lim _{\overline{\gamma_{1} \rightarrow \infty}} \bar{\gamma}_{1}^{2} \cdot P_{e}=3 /\left(8 \cdot \sigma_{0}\right) .
$$

Comparing (10) with (6), we find now that $d=2$ and $\mathcal{E}\left(\sigma_{0}\right)=\log _{10}\left(3 /\left(8 \sigma_{0}\right)\right)$. 


\subsection{Asymptotic BER for QCP}

For the computation of BER, we write the formula (5) as

$$
\begin{gathered}
P_{e}=\int_{\mathbb{R}^{3}} P_{\bmod }\left(z\left(\gamma_{1,1}, \gamma_{2,1}, \varphi\right)\right) f\left(\varphi, \gamma_{1,2}, \gamma_{2,1}\right) \\
d \gamma_{1,1} d \gamma_{2,1} d \varphi,
\end{gathered}
$$

where $\varphi=\psi_{2,1}-\psi_{1,1}+\hat{\phi}_{n}$. Since random variables $\varphi$, $\gamma_{1,1}$, and $\gamma_{2,1}$ are independent, we have $f\left(\varphi, \gamma_{1,1}, \gamma_{2,1}\right)=$ $U_{N}(\varphi) f\left(\gamma_{1,1}\right) f\left(\gamma_{2,1}\right)$, where $U_{N}$ refers to the uniform distribution in the interval $\left(-\pi / 2^{N_{w}}, \pi / 2^{N_{w}}\right)$ and $f\left(\gamma_{m, 1}\right)=$ $e^{-\gamma_{m, 1} / \bar{\gamma}_{m}} / \bar{\gamma}_{m}, m=1,2$. Let us substitute $\gamma_{1,1}=y$ and $\gamma_{2,1}=t y$. Then $z=y\left|\nu_{1}+v_{2} \sqrt{t} e^{j \varphi}\right|^{2}$, and we can write the BER of QCP in the form

$$
P_{e}=\frac{2^{N_{w}}}{\pi} \int_{0}^{\pi / 2^{N_{w}}} \int_{0}^{\infty} \frac{\mathcal{I}(t, \varphi) d t d \varphi}{\bar{\gamma}_{1} \bar{\gamma}_{2}\left|v_{1}+v_{2} \sqrt{t} e^{j \varphi}\right|^{4}}
$$

where for $c(t, \varphi)=\left(1 / \bar{\gamma}_{1}+t / \bar{\gamma}_{2}\right) /\left|v_{1}+v_{2} \sqrt{t} e^{j \varphi}\right|^{2}$,

$$
\mathcal{I}(t, \varphi)=\frac{1}{2} \int_{0}^{\infty} \operatorname{erfc}(\sqrt{\eta}) \eta e^{-c(t, \varphi) \eta} d \eta .
$$

In Appendix 1, we have shown that $\lim _{\bar{\gamma}_{1} \rightarrow \infty} \mathcal{I}(t, \varphi)=$ $3 / 16$. Using this result and (12), we obtain

$$
\lim _{\overline{\gamma_{1} \rightarrow \infty}} \bar{\gamma}_{1}^{2} \cdot P_{e}=3 /\left(16 \sigma_{0}\right) \cdot A_{N_{w}}\left(v_{1}, v_{2}\right)
$$

where

$$
A_{N_{w}}\left(v_{1}, v_{2}\right)=\frac{2^{N_{w}}}{\pi} \int_{0}^{\pi / 2^{N_{w}}} \int_{0}^{\infty} \frac{d t d \varphi}{\left|v_{1}+v_{2} \sqrt{t} e^{j \varphi}\right|^{4}}
$$

Furthermore, utilizing equality (3.252.4) from [27], we achieve a closed-form expression

$$
\begin{aligned}
& A_{N_{w}}\left(v_{1}, v_{2}\right)= \\
& \frac{1}{2 v_{1}^{2} v_{2}^{2}}\left[\csc ^{2}\left(\frac{\pi}{2^{N_{w}}}\right)-\frac{2^{N_{w}}}{\pi} \cot \left(\frac{\pi}{2^{N_{w}}}\right)\right] .
\end{aligned}
$$

Let us compare (14) and (16) with the asymptotic BER in the case of full CSI in (8). We conclude that the degradation of asymptotic BER due to quantized channel information is characterized by a constant which depends on the number of phase bits and long-term transmit weights $v_{1}$ and $v_{2}$. Furthermore, by comparing (14) with (6), we find that $d=2$ and $\mathcal{E}\left(\sigma_{0}\right)=\log _{10}\left(3 /\left(16 \sigma_{0}\right) \cdot A_{N_{w}}\left(\nu_{1}, \nu_{2}\right)\right)$. Moreover, optimal asymptotic BER is achieved when $v_{1}=$ $v_{2}=\sqrt{1 / 2}$ irrespective of the value of $\sigma_{0}$ as can be deduced from (14) and (16).

\subsection{Asymptotic BER for OQCP}

In this case, the average BER is of the form

$$
\begin{gathered}
P_{e}=\int_{\mathbb{R}^{3}} P_{\bmod }\left(z\left(\gamma_{(1)}, \gamma_{(2)}, \varphi\right)\right) f\left(\varphi, \gamma_{(1)}, \gamma_{(2)}\right) \\
d \gamma_{(1)} d \gamma_{(2)} d \varphi,
\end{gathered}
$$

where $f\left(\varphi, \gamma_{(1)}, \gamma_{(2)}\right)=U_{N}(\varphi) f\left(\gamma_{(1)}, \gamma_{(2)}\right)$; channel gains $\gamma_{(1)}, \gamma_{(2)}$ are ordered; and $f\left(\gamma_{(1)}, \gamma_{(2)}\right)$ is the corresponding joint PDF. In Appendix 2, we have shown that (17) reduces to

$$
P_{e}=\frac{2^{N_{w}}}{\pi} \int_{0}^{\pi / 2^{N_{w}}} \int_{0}^{1} \frac{\left(\mathcal{I}_{1}(t, \varphi)+\mathcal{I}_{2}(t, \varphi)\right) d t d \varphi}{\bar{\gamma}_{1} \bar{\gamma}_{2}\left|v_{1}+v_{2} \sqrt{t} e^{j \varphi}\right|^{4}} .
$$

Similarly with (13), notations $\mathcal{I}_{1}(t, \varphi)$ and $\mathcal{I}_{2}(t, \varphi)$ refer to definite integrals for which $\lim _{\bar{\gamma}_{1} \rightarrow \infty} \mathcal{I}_{1}(t, \varphi)=$ $\lim _{\bar{\gamma}_{1} \rightarrow \infty} \mathcal{I}_{2}(t, \varphi)=3 / 16$, and we obtain

$$
\lim _{\bar{\gamma}_{1} \rightarrow \infty} \bar{\gamma}_{1}^{2} \cdot P_{e}=3 /\left(8 \sigma_{0}\right) \cdot B_{N w}\left(v_{1}, v_{2}\right),
$$

where

$$
B_{N w}\left(v_{1}, v_{2}\right)=\frac{2^{N_{w}}}{\pi} \int_{0}^{\pi / 2^{N_{w}}} \int_{0}^{1} \frac{d t d \varphi}{\left|v_{1}+v_{2} \sqrt{t} e^{j \varphi}\right|^{4}} .
$$

By changing variables, we find as expected that $B_{N w}\left(v_{1}, v_{2}\right)=A_{N w}\left(v_{1}, v_{2}\right) / 2$ when $v_{1}=v_{2}$ irrespective of power imbalance. Let us now compute $B_{N w}\left(v_{1}, v_{2}\right)$ when $v_{1} \neq v_{2}$. Exploiting the Taylor expansion of $(1+x)^{-2}$, we can write $B_{N w}\left(u_{1}, u_{2}\right)$ in the form

$$
\begin{aligned}
B_{N w}= & \frac{2^{N_{w}}}{\pi} \int_{0}^{\pi / 2^{N_{w}}} \int_{0}^{1} \frac{1}{\left(1+\frac{2 v_{1} v_{2} \sqrt{t} \cos \varphi}{v_{1}^{2}+v_{2}^{2} t}\right)^{2}} \frac{d t d \varphi}{\left(v_{1}^{2}+v_{2}^{2} t\right)^{2}} \\
= & \sum_{n=0}^{\infty} \frac{2^{N_{w}}(-1)^{n}(n+1)}{\pi} \int_{0}^{\pi / 2^{N_{w}}} \cos ^{n}(\varphi) d \varphi \\
& \int_{0}^{1} \frac{\left(2 v_{1} v_{2} \sqrt{t}\right)^{n}}{\left(v_{1}^{2}+v_{2}^{2} t\right)^{n+2}} d t .
\end{aligned}
$$

We solve the remaining integrals using equations (2.513.3), (2.513.4), and (3.194.1) of [27]. Then $B_{N w}\left(v_{1}, v_{2}\right)$ becomes

$$
\begin{gathered}
B_{N w}=\sum_{n=0}^{\infty} \frac{2^{\left(N_{w}+1\right)}(-2)^{n}(n+1) v_{2}^{n}}{\pi(n+2) v_{1}^{n+4}}\left[A_{n}+\frac{1}{2^{n-1}} \sum_{k=0}^{B_{n}}\right. \\
\left.\left(\begin{array}{l}
n \\
k
\end{array}\right) \frac{\sin \left(\frac{(n-2 k) \pi}{2^{N_{w}}}\right)}{n-2 k}\right]{ }_{2} F_{1}\left(n+2, \frac{n}{2}+1 ; \frac{n}{2}+2 ; \frac{-v_{2}^{2}}{v_{1}^{2}}\right),
\end{gathered}
$$

where ${ }_{2} F_{1}$ is the confluent hypergeometric function, $A_{n}=$ $\left(\begin{array}{c}n \\ n / 2\end{array}\right) \pi / 2^{N+n}$ and $B_{n}=n / 2-1$ for even $n$ and $A_{n}=0$ and $B_{n}=(n-1) / 2$ for odd $n$. We see from (19) and (6) that $d=2$ and $\mathcal{E}\left(\sigma_{0}\right)=\log _{10}\left(3 /\left(8 \sigma_{0}\right) \cdot B_{N w}\left(v_{1}, v_{2}\right)\right)$, for OQCP.

Before we close the asymptotic BER analysis, in Table 2, we revise results of $\mathcal{E}\left(\sigma_{0}\right)$ obtained for the CoMP techniques.

\subsection{Approximation for the BER of QCP and OQCP}

Let us now formulate BER expression in the low-tomoderate SNR region for both QCP and OQCP based on their asymptotic BER and expected SNR expressions. We approximate the SNR distribution $f_{z}$ of QCP and OQCP 
Table 2 Achieved results for $\mathcal{E}\left(\sigma_{0}\right)$

\begin{tabular}{ll}
\hline CoMP method & Result for $\mathcal{E}\left(\sigma_{0}\right)$ \\
\hline TSC & $\mathcal{E}\left(\sigma_{0}\right)=\log _{10}\left(3 /\left(8 \sigma_{0}\right)\right)$ \\
QCP & $\mathcal{E}\left(\sigma_{0}\right)=\log _{10}\left(3 /\left(16 \sigma_{0}\right) \cdot A_{N_{w}}\left(v_{1}, v_{2}\right)\right)$ \\
OQCP & $\mathcal{E}\left(\sigma_{0}\right)=\log _{10}\left(3 /\left(8 \sigma_{0}\right) \cdot B_{N w}\left(v_{1}, v_{2}\right)\right)$ \\
Full CSI & $\mathcal{E}\left(\sigma_{0}\right)=\log _{10}\left(3 /\left(16 \sigma_{0}\right)\right)$ \\
\hline
\end{tabular}

by using the distribution $f_{\tilde{z}}$ of variable $\tilde{z}=\xi_{1}+\xi_{2}$, where $\xi_{m}, m=1,2$ follow the exponential distribution $f_{m}(\xi)=$ $\exp \left(-\xi / \bar{\xi}_{m}\right)$. Here means $\bar{\xi}_{1}$ and $\bar{\xi}_{2}$ are selected such that the following requirements hold the following:

(i) The first moments of $\tilde{z}$ and $z$ are equal, i.e., $E\{\tilde{z}\}=$ $E\{z\}$.

(ii) The asymptotic BERs, computed using $f_{\tilde{Z}}$ and $f_{Z}$, are equal, i.e., following the results (14) and (19), we require

$$
\lim _{\bar{\xi}_{1} \rightarrow \infty} \bar{\xi}_{1}^{2} \tilde{P}_{e}=\lim _{\bar{\gamma}_{1} \rightarrow \infty} \bar{\gamma}_{1}^{2} P_{e}
$$

where $\tilde{P}_{e}=\int_{0}^{\infty} P_{\bmod }(z) f_{\tilde{z}}(z) d z$ refers to the BER approximation and $P_{e}=\int_{0}^{\infty} P_{\bmod }(z) f_{z}(z) d z$ is the BER of QCP/OQCP, and it is assumed that ratios $\bar{\xi}_{1} / \bar{\xi}_{2}$ and $\bar{\gamma}_{1} / \bar{\gamma}_{2}$ are both fixed and larger than one. Requirement (i) using (50) and (54) in [16] and $E\{\tilde{z}\}=\bar{\xi}_{1}+\bar{\xi}_{2}$ leads to formula

$$
\bar{\xi}_{1}+\bar{\xi}_{2}=\bar{\gamma}_{1} \mathcal{L},
$$

where

$$
\mathcal{L}=\frac{1}{2}\left[1+\sigma_{0}+\sqrt{\left(1-\sigma_{0}\right)^{2}+\frac{\sigma \pi^{2}}{4} \operatorname{sinc}\left(\frac{\pi}{2^{N_{w}}}\right)}\right],
$$

for QCP and

$$
\mathcal{L}=\frac{1}{2}\left[1+\sigma_{0}+\sqrt{\left(\frac{1+\sigma_{0}^{2}}{1+\sigma_{0}}\right)^{2}+\frac{\sigma \pi^{2}}{4} \operatorname{sinc}\left(\frac{\pi}{2^{N_{w}}}\right)}\right],
$$

for OQCP. Furthermore, requirement (ii) using the asymptotic BER formulas in (8), (14), and (19) provides

$$
\bar{\xi}_{1} / \bar{\xi}_{2}=\bar{\gamma}_{1} / \bar{\gamma}_{2} \cdot C_{N_{w}}\left(v_{1}, v_{2}\right),
$$

where $C_{N_{w}}\left(v_{1}, v_{2}\right)=A_{N_{w}}\left(v_{1}, v_{2}\right)$ for QCP and $C_{N_{w}}\left(v_{1}, v_{2}\right)=$ $2 B_{N_{w}}\left(v_{1}, v_{2}\right)$ for OQCP. After combining (23) and (26), we find that

$$
\bar{\xi}_{1}=\frac{C_{N_{w}} \cdot \mathcal{L} \cdot \bar{\gamma}_{1}}{\sigma_{0}+C_{N_{w}}}, \quad \bar{\xi}_{2}=\frac{\sigma_{0} \cdot \mathcal{L} \cdot \bar{\gamma}_{1}}{\sigma_{0}+C_{N_{w}}},
$$

where we have shortened notations by dropping out arguments of $C_{N_{w}}$. Then the BER approximation $\tilde{P}_{e}$ for QCP/OQCP is obtained by combining (27) and (7). After some elementary manipulations, we find that

$$
\begin{aligned}
P_{e}= & \frac{1}{2}\left[1-\frac{1}{1-\sigma_{0} / C_{N_{w}}} \sqrt{\frac{\mathcal{L} \bar{\gamma}_{1}}{1+\sigma_{0} / C_{N_{w}}+\mathcal{L} \bar{\gamma}_{1}}}\right. \\
& \left.\left(1-\frac{\sigma_{0}}{C_{N_{w}}} \sqrt{\frac{1+\sigma_{0} / C_{N_{w}}+\mathcal{L} \bar{\gamma}_{1}}{1+C_{N_{w}} / \sigma_{0}+\mathcal{L} \bar{\gamma}_{1}}}\right)\right] .
\end{aligned}
$$

We note that this formula is valid if $\sigma_{0}<C_{N_{w}}$. Yet, this is not a limitation since $A_{N_{w}}$ and $2 B_{N_{w}}$ are both larger than one. According to our knowledge, this approximation has been previously used only in [22].

\section{Validation and performance evaluations}

\subsection{Validation and performance results for $M=1$}

The asymptotic and approximate BER expressions presented in Section 3 are validated in Fig. 2. Markers refer to the simulated BER while dashed and solid curves refer to the analytical asymptotic and the approximate BERs, respectively. Results are presented for power balance and 6-dB imbalance cases assuming $N_{w}=3$ for both QCP and OQCP. For OQCP, we use the SNR maximizing long-term weights presented in [16]:

$v_{1,2}^{2}=\frac{1}{2}\left[1 \pm \frac{1+\sigma_{0}-\frac{2 \sigma_{0}}{1+\sigma_{0}}}{\sqrt{\left(1+\sigma_{0}-\frac{2 \sigma_{0}}{1+\sigma_{0}}\right)^{2}+\frac{\pi^{2} \sigma_{0}}{4} \operatorname{sinc}^{2}\left(\frac{\pi}{2^{N_{w}}}\right)}}\right]$,

and for QCP we set $v_{1}=v_{2}=\sqrt{1 / 2}$. As expected, we see that the CoMP techniques are negatively impacted when the power imbalance increases from 0 to $6 \mathrm{~dB}$.

The impact of channel power imbalance on the BER of the studied schemes is shown in Figs. 3 and 4 where average BER results are presented as a function of power imbalance assuming $\bar{\gamma}_{1}=10 \mathrm{~dB}$ and $\bar{\gamma}_{1}=15 \mathrm{~dB}$, respectively. The results are obtained for $N_{w}=3$, and note that QCP is plotted with equal weights (diamond marked) and SNR maximizing weights (circle marked) that are formulated in [16]:

$$
v_{1,2}^{2}=\frac{1}{2}\left[1 \pm \frac{1-\sigma_{0}}{\sqrt{\left(1-\sigma_{0}\right)^{2}+\frac{\pi^{2} \sigma_{0}}{4} \operatorname{sinc}^{2}\left(\frac{\pi}{2^{N_{\mathrm{rp}}}}\right)}}\right] .
$$

We observe from both figures that OQCP performs close to the case where full CSI is applied irrespective of the value of power imbalance. Interestingly, we also see that TSC outperforms QCP applying the SNR maximizing long-term weights when there is large power imbalance. As can be seen from Figs. 3 and 4, TSC outperforms the QCP after a power imbalance value of around -6 and $-5 \mathrm{~dB}$ when $\bar{\gamma}_{1}=10 \mathrm{~dB}$ and $\bar{\gamma}_{1}=15 \mathrm{~dB}$, respectively. On the other hand, QCP applying the asymptotic BER minimizing weights $\left(v_{1}=v_{2}\right)$ performs close to OQCP at a large imbalance. 

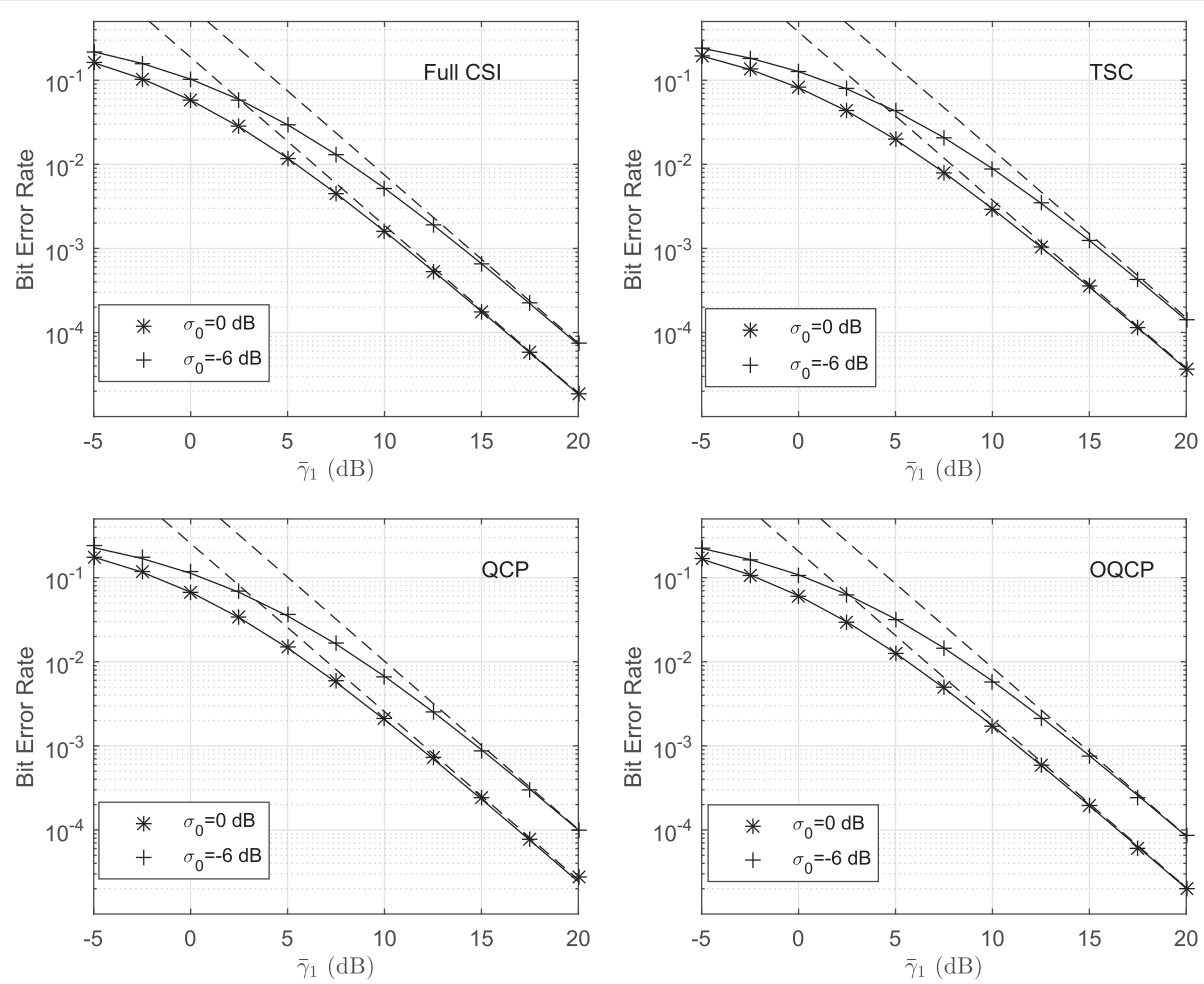

Fig. 2 Bit error rate as a function of $\bar{\gamma}_{1}$ when $\sigma_{0}=0 \mathrm{~dB}$ and $\sigma_{0}=6 \mathrm{~dB}$. Dashed curves refer to the analytical asymptotic BER results; solid curves refer to the approximate BER results; and markers refer to the simulated BER results

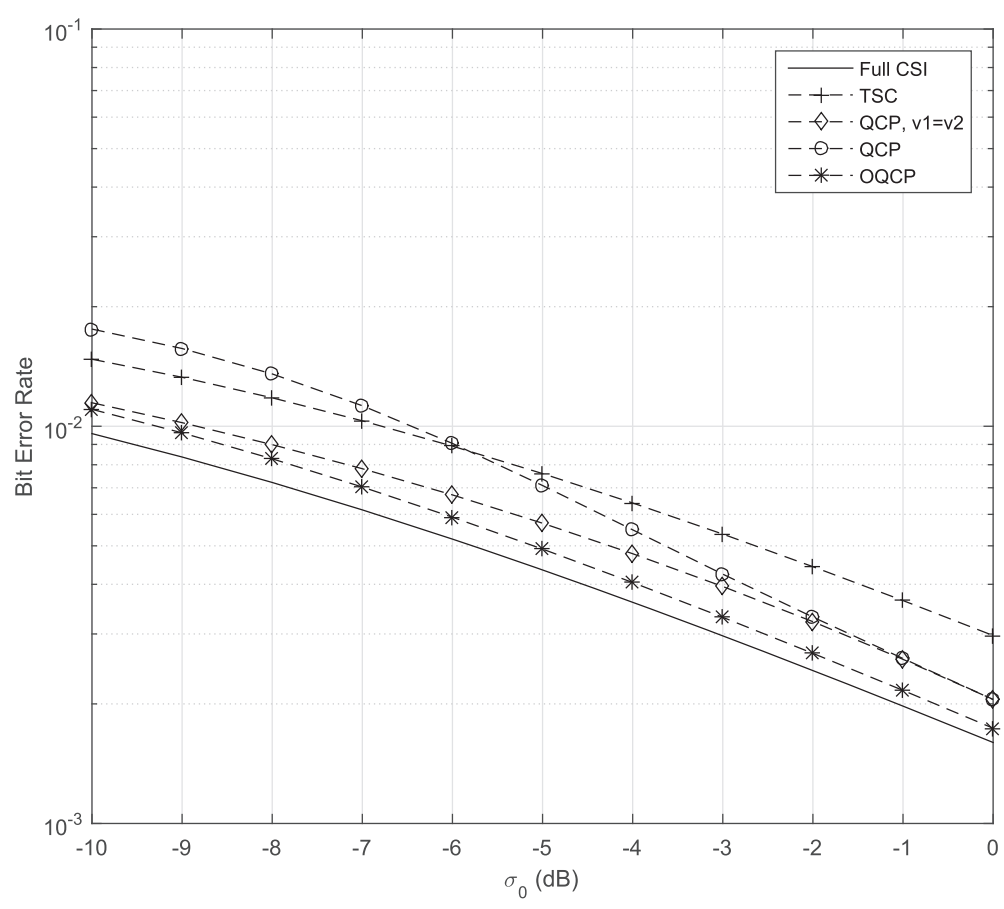

Fig. 3 Analytical BER as a function of $\sigma_{0}$ for QCP, OQCP, TSC, and full CSI schemes when $\bar{\gamma}_{1}=10 \mathrm{~dB}$ 


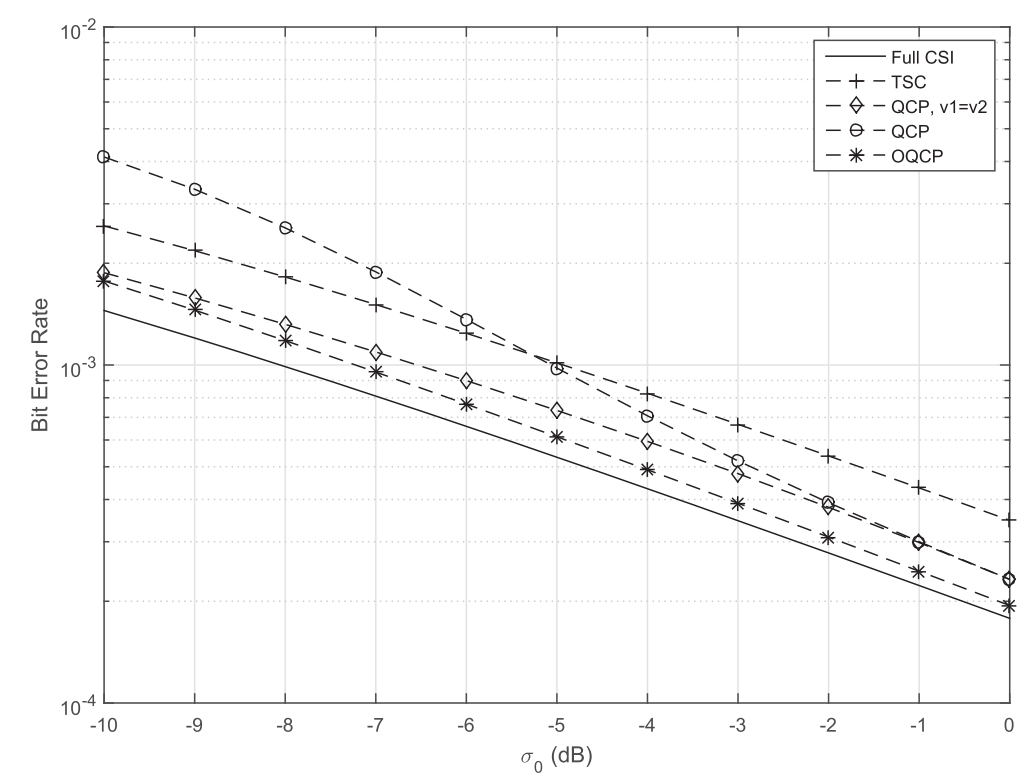

Fig. 4 Analytical BER as a function of $\sigma_{0}$ for QCP, OQCP, TSC, and full CSI schemes when $\bar{\gamma}_{1}=15 \mathrm{~dB}$

\subsection{Numerical results for $M>1$}

To observe impacts of using more numbers of transmit antennas in BSs, we present simulated BER results in Fig. 5 when two antennas are applied in each BS. The results are obtained for $\bar{\gamma}_{1}=10 \mathrm{~dB}, N_{w}=3$, and $N_{u}=2$. We see from the figure that unlike the $M=1$ case, QCP applying SNR-maximizing long-term weights outperforms the TSC throughout the power imbalance range. This comes from the diversity gain achieved from the multiple antennas utilized in the BSs. More performance improvement is achieved by the QCP when more numbers of antennas are employed by the BSs as illustrated in Fig. 6 where we depict the BER results for $M=4, \bar{\gamma}_{1}=3 \mathrm{~dB}, N_{w}=3$, and $N_{u}=2$. In this case, QCP applying SNR-maximizing weights performs very close to OQCP, particularly for large power imbalance.

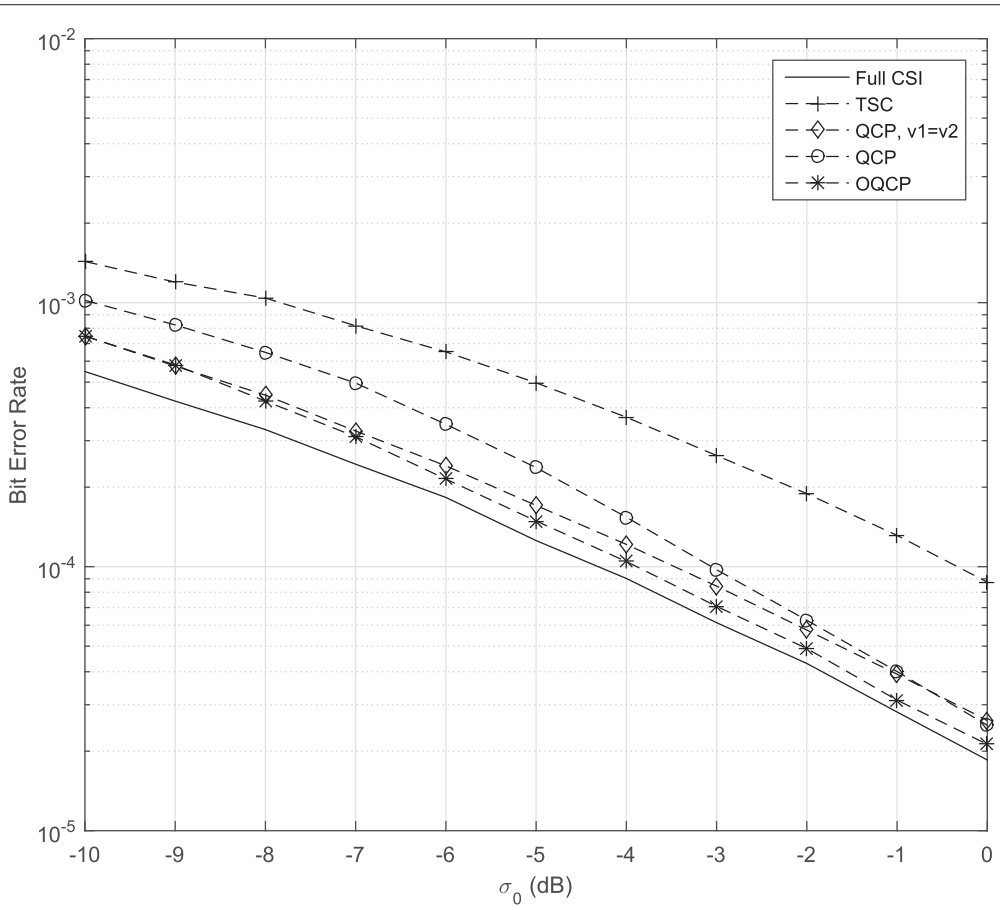

Fig. 5 Simulated BER as a function of $\sigma_{0}$ for QCP, OQCP, TSC, and full CSI schemes when $M=2$ and $\bar{\gamma}_{1}=10 \mathrm{~dB}$ 


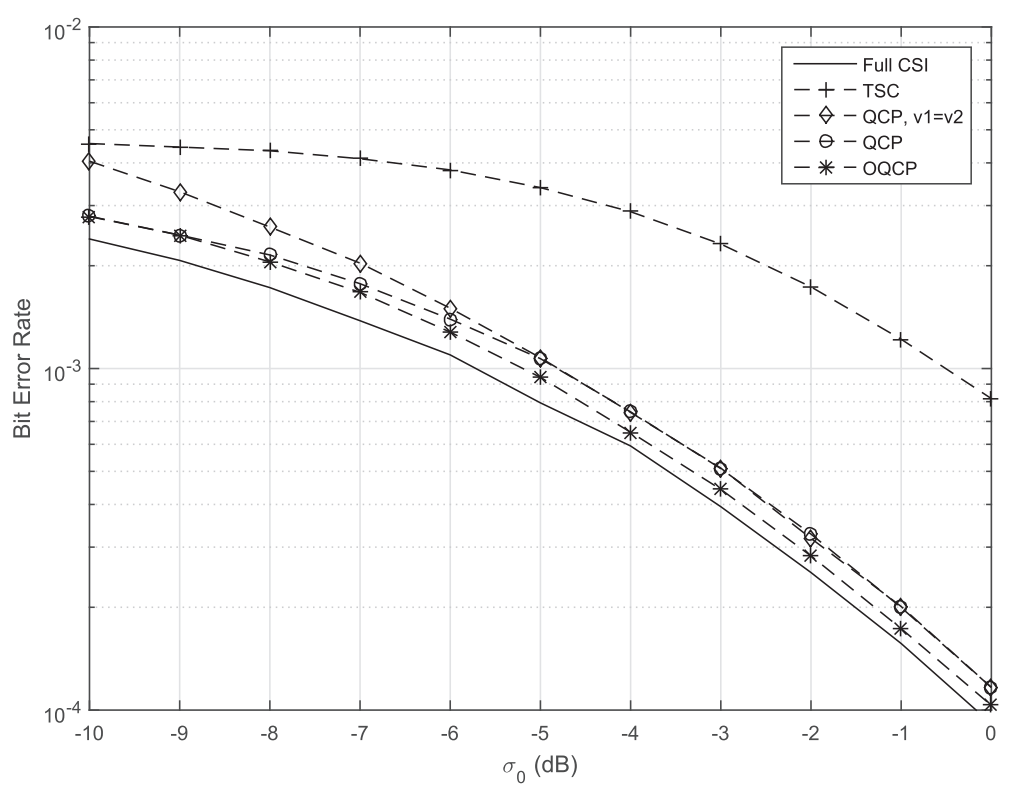

Fig. 6 Simulated BER as a function of $\sigma_{0}$ for QCP, OQCP, TSC, and full CSI schemes when $M=4$ and $\bar{\gamma}_{1}=3 \mathrm{~dB}$

\section{Conclusions}

We studied the impact of mean channel power imbalance on CoMP transmission. Specifically, we investigated CoMP techniques called QCP and OQCP where the former scheme applies quantized channel phase feedback while the latter technique applies order information in addition to the quantized phase at the transmitter side. We derived closed-form expressions for the asymptotic and the approximate BERs and verified analytical results using numerical simulations when base stations apply a singletransmit antenna. For complete understanding of channel power imbalance impacts, we also presented numerical analysis for cases where base stations employ more than one transmit antenna. Even with few feedback bits and presence of channel power imbalance, the OQCP provides performance that is very close to the performance achieved with the case where full CSI is applied. Unlike the average capacity performance, presented in [16], using SNR maximizing long-term amplitude weights for QCP under power imbalance worsens the BER performance when a single-transmit antenna is utilized in BSs due to limited diversity gain. This is not the case when BSs apply more than one diversity antenna. In future work, analytical analysis will be extended for general $M$ and other modulation schemes.

\section{Appendix 1}

We show here that $\lim _{c \rightarrow 0} \frac{1}{2} \int_{0}^{\infty} \eta e^{-c \eta} \operatorname{erfc}(\sqrt{\eta}) d \eta=$ $3 / 16$. Utilizing equations (7.1.2) and (7.4.19) in [28], the integral can be written as

$$
\begin{aligned}
& \int_{0}^{\infty} \eta e^{-c \eta} \operatorname{erfc}(\sqrt{\eta}) d \eta= \\
& -\frac{d}{d c} \int_{0}^{\infty} e^{-c \eta} \operatorname{erfc}(\sqrt{\eta}) d \eta=-\frac{d}{d c}\left[\frac{1}{c}\left(1-\sqrt{\frac{1}{1+c}}\right)\right]
\end{aligned}
$$

Using Taylor expansion of $(1+c)^{-1 / 2}$, we obtain

$$
\frac{1}{c}\left(1-\sqrt{\frac{1}{1+c}}\right)=\sum_{k=0}^{\infty} \frac{(-1)^{k}(2 k+1) !}{2^{2 k+1} k !(k+1) !} c^{k}=\sum_{k=0}^{\infty} D_{k} c^{k},
$$

where $D_{k}=\frac{(-1)^{k}(2 k+1) !}{2^{2 k+1} k !(k+1) !}$. Combining the last two formulas yields the desired result.

\section{Appendix 2}

Our goal is to prove (18). Applying basic theory of order statistic, we find the joint $\operatorname{PDF} f\left(\gamma_{(1)}, \gamma_{(2)}\right)$ as

$$
f\left(\gamma_{(1)}, \gamma_{(2)}\right)=\frac{1}{\gamma_{(1)} \gamma_{(2)}}\left[e^{-\left(\frac{\gamma_{(2)}}{\gamma_{1}}+\frac{\gamma_{(1)}}{\gamma_{2}}\right)}+e^{-\left(\frac{\gamma_{(1)}}{\gamma_{1}}+\frac{\gamma_{(2)}}{\gamma_{2}}\right)}\right] .
$$

With this joint PDF, we can easily deduce from (17) that

$$
\begin{aligned}
\bar{P}_{e}= & \frac{2^{N_{w}}}{2 \pi} \int_{0}^{\frac{\pi}{2^{N_{W}}}} \int_{0}^{\gamma_{(1)}} \int_{0}^{\infty} \operatorname{erfc}\left(\left|v_{1} \sqrt{\gamma_{(1)}}+v_{2} \sqrt{\gamma_{(2)}} e^{j \varphi}\right|\right) \\
& \frac{e^{-\left(\frac{\gamma_{(2)}}{\gamma_{1}}+\frac{\gamma_{(1)}}{\gamma_{2}}\right)}+e^{-\left(\frac{\gamma_{(1)}}{\gamma_{1}}+\frac{\gamma_{(2)}}{\gamma_{2}}\right)}}{\bar{\gamma}_{1} \bar{\gamma}_{2}} d \gamma_{(1)} d \gamma_{(2)} d \varphi .
\end{aligned}
$$


First, we set $\gamma_{1,1}=y, \gamma_{2,1}=t y$, and then, we substitute

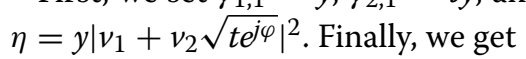

$$
\bar{P}_{e}=\frac{2^{N_{w}}}{\pi} \int_{0}^{\frac{\pi}{2^{N_{W}}}} \int_{0}^{1} \frac{\left(I_{1}(t, \varphi)+I_{2}(t, \varphi)\right) d t d \varphi}{\bar{\gamma}_{1} \bar{\gamma}_{2}\left|v_{1}+v_{2} \sqrt{t} e^{j \varphi}\right|^{4}},
$$

where $I_{1}(t, \varphi)$ and $I_{2}(t, \varphi)$ refer to the integrals

$$
\begin{aligned}
& I_{1}(t, \varphi)=\frac{1}{2} \int_{0}^{\infty} \operatorname{erfc}(\sqrt{\eta}) \eta e^{-c \eta} d \eta, c=\frac{\frac{t}{\bar{\gamma}_{1}}+\frac{1}{\bar{\gamma}_{2}}}{\left|\nu_{1}+v_{2} \sqrt{t} e^{j \varphi}\right|^{2}}, \\
& I_{1}(t, \varphi)=\frac{1}{2} \int_{0}^{\infty} \operatorname{erfc}(\sqrt{\eta}) \eta e^{-d \eta} d \eta, d=\frac{\frac{1}{\overline{\gamma_{1}}}+\frac{t}{\overline{\gamma_{2}}}}{\left|\nu_{1}+v_{2} \sqrt{t} e^{j \varphi}\right|^{2}} .
\end{aligned}
$$

\section{Acknowledgements}

This material is based on works supported in part by the academy of Finland under grant 284634 and the European institute for innovation and technology under grant 602954.

\section{Competing interests}

The authors declare that they have no competing interests.

\section{Author details}

${ }^{1}$ School of Electrical Engineering, Aalto University, P.O.Box 13000, FI-00076 Aalto, Espoo, Finland. ${ }^{2}$ Department of Electrical and Computer Engineering, University of California, Davis, USA.

Received: 1 March 2016 Accepted: 13 August 2016

Published online: 06 September 2016

\section{References}

1. N Alliance, CoMP evaluation and enhancement. Ran evolution project deliverable (2015)

2. MK Karakayali, GJ Foschini, RA Valenzuela, Network coordination for spectrally efficient communications in cellular systems. IEEE Wireless Commun. Mag. 13(4), 56-61 (2006)

3. R Irmer, H Droste, P Marsch, M Grieger, G Fettweis, S Brueck, H-P Mayer, L Thiele, $V$ Jungnickel, Coordinated multipoint: concepts, performance, and field trial results. IEEE Commun. Mag. 49(2), 102-111 (2011). doi:10.1109/MCOM.2011.5706317

4. X Tao, XXu, Q Cui, An overview of cooperative communications. IEEE Commun. Mag. 50(6), 65-71 (2012). doi:10.1109/MCOM.2012.6211487

5. H Dahrouj, W Yu, Coordinated beamforming for the multicell multi-antenna wireless system. IEEE Trans. Wireless Commun. 9(5), 1748-1759 (2010). doi:10.1109/TWC.2010.05.090936

6. J Zhao, TQS Quek, Z Lei, Coordinated multipoint transmission with limited backhaul data transfer. IEEE Trans. Wireless Commun. 12(6), 2762-2775 (2013). doi:10.1109/TWC.2013.050613.120825

7. 3GPP, Coordinated multi-point operation for LTE physical layer aspects. 3GPP Technical Report (2011). 36.819, Ver. 11.0.0

8. 3GPP, Coordinated multi-point operation for LTE with non-ideal backhaul. 3GPP Technical Report (2013). 36.874, Ver. 12.0.0

9. DJ Love, RW Heath, VKN Lau, D Gesbert, BD Rao, M Andrews, An overview of limited feedback in wireless communication systems. IEEE J. Sel. Areas Commun. 26(8), 1341-1365 (2008). doi:10.1109/JSAC.2008.081002

10. N Jindal, MIMO broadcast channels with finite-rate feedback. IEEE Trans. Inf. Theory. 52(11), 5045-5060 (2006). doi:10.1109/TIT.2006.883550

11. T Yoo, N Jindal, A Goldsmith, Multi-antenna downlink channels with limited feedback and user selection. IEEE J. Sel. Areas Commun. 25(7), 1478-1491 (2007). doi:10.1109/JSAC.2007.070920

12. J Hämäläinen, R Wichman, AA Dowhuszko, G Corral-Briones, Capacity of generalized UTRA FDD closed-loop transmit diversity modes. Wireless Personal Commun., 1-18 (2009)

13. HC Papadopoulos, C-EW Sundberg, Space-time codes for MIMO systems with non-collocated transmit antennas. IEEE J. Sel. Areas Commun. 26(6), 927-937 (2008). doi:10.1109/JSAC.2008.080809

14. 3GPP, Spatial channel model for Multiple Input Multiple Output (MIMO) simulations. 3GPP Technical Specification (2015). 25.996, Ver. 13.0.0
15. 3GPP, Base Station (BS) radio transmission and reception (FDD). 3GPP Technical Specification (2011). 25.104, Ver. 10.3.0

16. BB Haile, AA Dowhuszko, J Hamalainen, R Wichman, Z Ding, On performance loss of some CoMP techniques under channel power imbalance and limited feedback. IEEE Trans. Wireless Commun. 14(8), 4469-4481 (2015). doi:10.1109/TWC.2015.2421898

17. J Hamalainen, R Wichman, in Proc. IEEE Global Telecommun. Conf. On correlations between dual-polarized base station antennas, vol. 3 (IEEE, US, 2003), pp. 1664-16683

18. J Hämäläinen, R Wichman, in Proc. IEEE Int. Symp. on Personal, Indoor and Mobile Radio Commun. Performance analysis of closed-loop transmit diversity in the presence of feedback errors, vol. 5 (IEEE, US, 2002), pp. 2297-2301

19. 3GPP, Physical layer procedures (FDD). 3GPP Technical Specification (2013). 25.214, Ver. 13.0.0

20. 3GPP, Physical Channels and Modulation. 3GPP Technical Specification (2015). 36.211, Ver. 12.7.0

21. J Hämäläinen, R Wichman, in Proc. Asilomar Conf. on Signals, Systems and Computers. Closed-loop transmit diversity for FDD WCDMA systems, vol. 1 (IEEE, US, 2000), pp. 111-115. doi:10.1109/ACSSC.2000.910927

22. J Hämäläinen, R Wichman, in Proc. IEEE Global Telecommun. Conf. Asymptotic bit error probabilities of some closed-loop transmit diversity schemes, vol. 1 (IEEE, US, 2002), pp. 360-364

23. A Papoulis, Probability, Random Variables, and Stochastic Processes. (McGraw-Hill, New York, 1984)

24. A Goldsmith, Wireless Communications. (Cambridge University Press, New York, 2005)

25. AF Coskun, O Kucur, Performance analysis of joint single transmit and receive antenna selection in non-identical nakagami-m fading channels. IET Commun. 5(14), 1947-1953 (2011). doi:10.1049/iet-com.2010.0719

26. JM Romero-Jerez, AJ Goldsmith, Performance of multichannel reception with transmit antenna selection in arbitrarily distributed nagakami fading channels. IEEE Trans. Wireless Commun. 8(4), 2006-2013 (2009). doi:10.1109/TWC.2009.080333

27. IS Gradshteyn, IM Ryzhik, Table of Integrals, Series, and Products, 7th edn. (Elsevier/Academic Press, Amsterdam, 2007), p. 1171

28. M Abramowitz, IA Stegun, Handbook of Mathematical Functions: with Formulas, Graphs, and Mathematical Tables. (Dover Publications, New York, 1972), p. 1046

\section{Submit your manuscript to a SpringerOpen ${ }^{\circ}$ journal and benefit from:}

- Convenient online submission

- Rigorous peer review

- Immediate publication on acceptance

- Open access: articles freely available online

- High visibility within the field

- Retaining the copyright to your article

Submit your next manuscript at $\gg$ springeropen.com 